Article

\title{
Numerical Research of the Effect of Surface Biomimetic Features on the Efficiency of Tidal Turbine Blades
}

\author{
Wenxian Yang*(D), Theodoros Alexandridis and Wenye Tian \\ School of Engineering, Newcastle University, Newcastle upon Tyne NE1 7RU, UK; \\ thodoris.alexandridis@gmail.com (T.A.); tianwenye@hotmail.com (W.T.) \\ * Correspondence: wenxian.yang@ncl.ac.uk; Tel.: +44-191-208-6171
}

Received: 12 March 2018; Accepted: 19 April 2018; Published: 21 April 2018

\begin{abstract}
Horizontal-axis axial flow tidal current turbine is regularly used to exploit the kinematic energy in tidal currents. However, the scaling up of tidal current turbine is very difficult. This is because strong tidal current only exists in the underwater region close to water surface, which implies that scaling up by enlarging rotor size is not always applicable to tidal current turbines. Hence, scaling up by improving the energy capture efficiency of the tidal turbine blade becomes a plausible choice. For this reason, apart from the numerous researches based on conventional aerodynamic and hydrodynamic theories, improving efficiency by biomimetic method is attracting increasing interest in recent years. It has been proved that leading-edge tubercles have positive contribution to improving the efficiency of tidal turbine blade. However, leading-edge tubercles can be made on blade only in the manufacturing process, as the post-production of them is quite difficult. Thus, how to improve the energy capture efficiency of the existing blades becomes a challenging issue. To address this issue, numerical research of the effect of surface biomimetic features on blade efficiency is conducted in this paper. For the sake of simplicity, surface bumps are investigated in this preliminary research in order to obtain a basic understanding of the effect of surface biomimetic features. In the research, the influences of surface bumps on blade surface pressure and the ratio of lift to drag forces are investigated in different bump array scenarios and at different tidal current speeds and the angles of attack. The calculation results have shown that surface bumps do improve the ratio of lift to drag forces of the blade in spite of their array arrangement, the angle of attack and tidal current speed. This suggests that the energy capture efficiency of both new and existing blades can be further improved if appropriate biomimetic features are deployed on the blade surfaces.
\end{abstract}

Keywords: tidal current turbine; biomimetics; blade; energy capture efficiency

\section{Introduction}

Due to the periodic motion of the moon and sun relative to the earth, regular movements of the oceans and seas are formed. The vertical rise and fall of water in these movements is known as oceanic tide, which is always accompanied by horizontal incoming and outgoing flow of water in bays, harbors, estuaries and straits. These horizontal flows are the so-called tidal currents [1]. It is predicted that the global tidal current energy is approximately $3 \mathrm{TW}$, of which about $1 \mathrm{TW}$ is located in shallow waters and exploitable via developing economically viable projects [2]. For example, 106 locations with strong tidal current have been identified in Europe. The majority of them are located in the British Isles and English Channel and have tidal current speeds exceeding 2-2.5 m/s [3]. Moreover, the potential of the tidal current in these areas may increase when water flow is constrained or funneled by local topography, such as marrow straits, headlands, and anywhere the water depth is relatively shallow. 
This will further benefit the harvest of the kinematic energy carried by the tidal currents. Obviously, tidal current is one of the most valuable renewable energy resources worthy to explore. It is believed that the conversion of these widely available renewable resources to electric power will play a vital role in meeting the rising energy demand, mitigating climate change, and diversifying energy supply. Moreover, as opposed to wind resource, tidal current resource is more stable and predictable attributed to the regular motion of the moon and sun relative to the earth.

In order to explore tidal current resources, much effort has been made across the world although there is still no large commercial tidal current array operating in the world today except a few prototypes that are undergoing test. However, thanks to these early efforts, a variety of designs of tidal current conversion systems have been developed, such as horizontal-axis axial flow turbine, vertical-axis cross flow turbine, reciprocating device, ducted turbines, tidal kite, and so on [1,3]. Among these designs, horizontal-axis axial flow turbine, a concept of stream device that is broadly similar to a 'submerged wind turbine', is relatively more mature in technology because many proven technologies that were well developed for wind turbines can be applied to the design of horizontal-axis tidal turbines. Accordingly, nowadays approximately $\frac{3}{4}$ of all research \& development (R\&D) investments are focused on horizontal-axis tidal current turbines versus on other designs [4]. However, different from the scaling up of wind turbines that can be realized by various approaches, the methods that can be adopted for scaling up tidal current turbine are quite few. There are numerous reasons for this, but one of the major reasons is due to the specific velocity profile of tidal current along underwater distance. In other words, strong tidal current can be observed only in the underwater region where is close to water surface. The kinematic energy of tidal current will decrease gradually with the increase of the underwater distance due to the gradually reduced tidal current speed [5]. This means that once the capacity of a tidal turbine is raised up to a certain value, further scaling up of it by size enlarging approach will become very difficult. For this reason, to improve the energy capture efficiency of rotor blade becomes a potential way to further scaling up the horizontal-axis tidal turbines.

In order to achieve an efficient turbine blade, a number of blade design methods and theories have been developed for optimizing the geometries of the blade, for example computing the thickness and chord length of the blade according to Betz limit, local fluid velocities and airfoil lift [6-9]. A brief review of these methods and theories can be found from [10]. They will not be repeated once again in this paper in order to keep a concise context. In addition to these conventional blade design methods that were developed based on the fundamental theory of hydro- and aero-dynamics, the boom of designing efficient blade using biomimetic methods is also seen in recent years [11-16]. Biomimetic techniques take inspiration from natural designs. They imitate the models, systems, and elements of nature for solving the complex and difficult problems met in engineering practice. Nowadays, they have been applied in a variety of fields. For example, vortex generators have been proved valid in achieving various purposes of passive flow control [17-20]. The application of vortex generators either delay or prevent the separation of the flow from structure surface and therefore decrease the roughness sensitivity of the structure of interest. The biomimetic technique was also employed to improve the energy capture efficiency of tidal turbine blades. A classic example is the humpback whale turbine blades, which adapt the whale's flipper and the flipper leading-edge tubercles to the design of blades [11,12]. Moreover, the positive contribution of the leading-edge tubercles to improving the energy capture efficiency of the blade has been validated in many numerical and experimental researches [13-15]. However, leading-edge tubercles can be made on the blade only in manufacturing process. The post-production of these tubercles on the leading edge of the blade will become very difficult. This implies that the leading-edge-tubercles are only applicable to new blades. They are quite difficult to be applied to the existing blades. Then, how to use biomimetic technique to improve the energy capture efficiency of the existing blades? To date, this is still an issue that has not been successfully solved. In order to fill this gap of technology, a new attempt is made in this paper to improve the efficiency of an existing blade by applying surface biomimetic features. In contrast to adding tubercles on leading-edge, the biomimetic features are deployed on the surface of the blade 
in this research. This is because biomimetic features can be easily made on the surface of the blade even though the blade has been produced. For the sake of simplicity, only round-shaped bumps are investigated in this research. Their influences on the blade surface pressure, the ratio of lift to drag forces, and torque created by every blade are numerically investigated under different conditions of bump array arrangement, angle of attack, and tidal current speed. The numerical simulation of the biomimetic blades and the rotor of tidal current turbine is realized with the aid of ANSYS CFX (Version R15.0, ANSYS Inc., Canonsburg, PA, USA). Through performing the numerical calculations, a number of valuable conclusions have been obtained from this numerical research. The details are reported below.

\section{Numerical Model of the Biomimetic Blade}

In the following, the scaled S-series airfoils developed by American National Renewable Energy Laboratory (NREL) [14], i.e., S818, S825 and S826, are employed to design the numerical model of the tidal turbine blade. Along the spanwise direction of the blade from root to tip, the blade begins with a circular section first. Then, the circular section gradually transitions to the S818 airfoils of different thicknesses and chord lengths. At the beginning of the middle section of the blade, the S818 transitions to the S825. Finally, the S825 transitions to the S826 at the beginning of the tip section of the blade. Considering the $41 \mathrm{~m}$ long wind turbine blade designed by Cornell University also adopts the similar design [21], the tidal turbine blade is designed in this paper by referring to the data provided by Cornell University. The detailed geometries of the blade, including twist angles, chord lengths and span distances, are listed in Table 1 . Where, ' $r / R$ ' and ' $c / R$ ' respectively refer to the normalized radial positions and normalized chord lengths of the blade elements. Considering the blades used in tidal current turbines are usually much shorter than wind turbine blades, the length of the blade is scaled down to $7 \mathrm{~m}$ in this research.

Table 1. Geometries of blade elements.

\begin{tabular}{ccccccc}
\hline Element & $\mathbf{r} / \mathbf{R}$ & Twist (degree) & $\mathbf{c} / \mathbf{R}$ & Span $(\mathbf{m})$ & Chord $(\mathbf{m})$ & Aerofoil \\
\hline 1 & 0.075 & 42 & 0.0614 & 0.525 & 0.4298 & \\
2 & 0.125 & 32 & 0.06826 & 0.875 & 0.47782 & \\
3 & 0.175 & 23 & 0.07452 & 1.225 & 0.52164 & \\
4 & 0.225 & 15 & 0.07782 & 1.575 & 0.54474 & S818 \\
5 & 0.275 & 11.5 & 0.07543 & 1.925 & 0.52801 & \\
6 & 0.325 & 8.2 & 0.07188 & 2.275 & 0.50316 & \\
7 & 0.375 & 7 & 0.06832 & 2.625 & 0.47824 & \\
8 & 0.425 & 6 & 0.06479 & 2.975 & 0.45353 & \\
9 & 0.475 & 5 & 0.06126 & 3.325 & 0.42882 & \\
10 & 0.525 & 4 & 0.05771 & 3.675 & 0.40397 & \\
11 & 0.575 & 4.15 & 0.05415 & 4.025 & 0.37905 & \\
12 & 0.625 & 3.85 & 0.05062 & 4.375 & 0.35434 & \\
13 & 0.675 & 3.25 & 0.04707 & 4.725 & 0.32949 & \\
14 & 0.725 & 2.75 & 0.0436 & 5.075 & 0.3052 & \\
15 & 0.775 & 1.25 & 0.04024 & 5.425 & 0.28168 & \\
16 & 0.825 & 0.75 & 0.03704 & 5.775 & 0.25928 & \\
\hline 17 & 0.875 & 0.55 & 0.03385 & 6.125 & 0.23695 & \\
18 & 0.925 & 0.85 & 0.03066 & 6.475 & 0.21462 & S826 \\
19 & 0.975 & 0.05 & 0.02747 & 6.825 & 0.19229 & \\
20 & 1 & 0 & 0.02424 & 7.000 & 0.16968 & \\
\hline
\end{tabular}

Based on the data listed in Table 1, the numerical model of the blade is developed in ANSYS CFX and it is shown in Figure 1. 


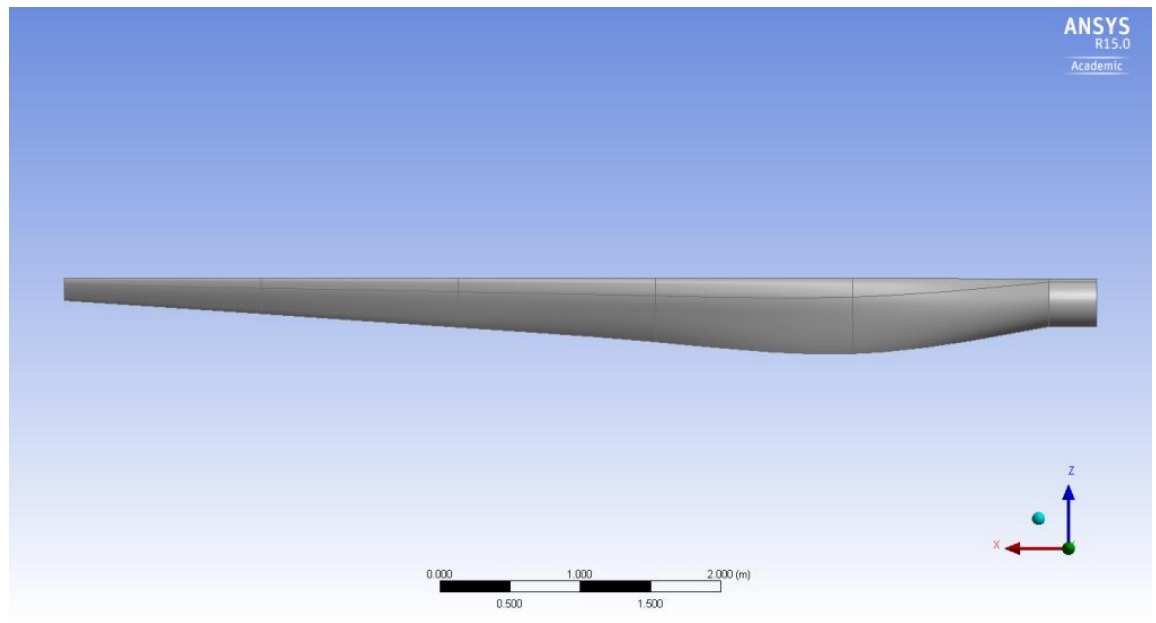

Figure 1. Numerical model of the blade.

Then, biomimetic features, i.e., surface bumps are created on the surface of the blade with the aid of the 'revolve function' that is available in ANSYS workspace. An illustrative example of the surface bumps is shown in Figure 2. As this research is mainly focused on investigating the influence of the presence of surface biomimetic features on the energy capture efficiency of the blade, the investigation of the size effect of the bumps is beyond the scope of this paper. Therefore, a constant size of the bumps, i.e., $5 \mathrm{~mm}$, is adopted in all numerical calculations described the following.

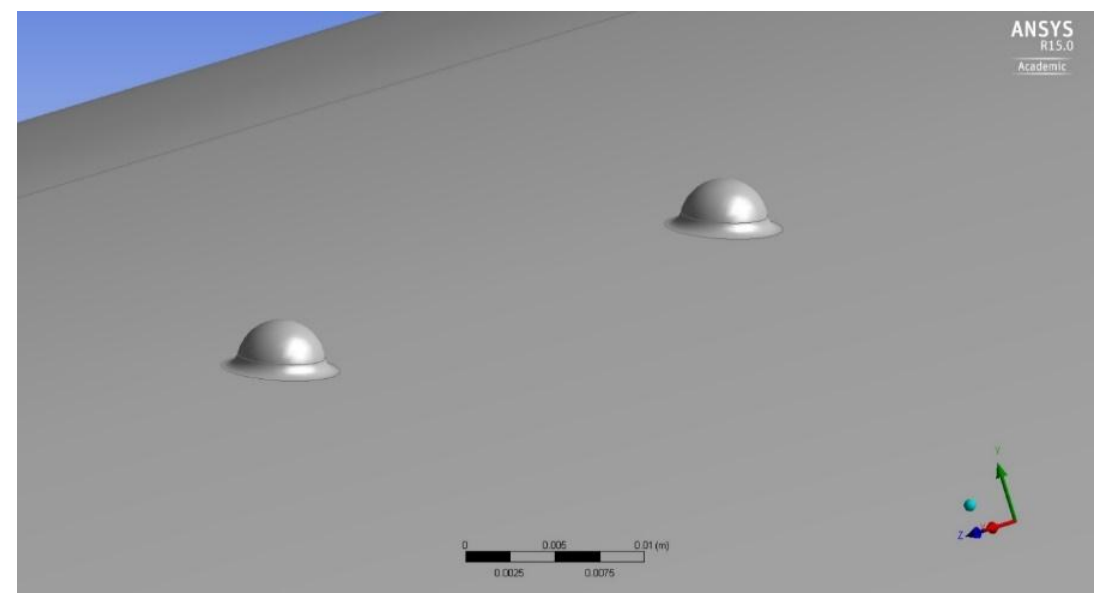

Figure 2. Illustration of the bumps on blade surface.

In order to systematically investigate the influences of the surface bumps on the energy capture efficiency of the blade, the following four bump array arrangement scenarios are considered in the calculations. They are:

- Scenario 1-26 bumps created near the leading-edge of the tip section of the blade;

- Scenario 2-93 bumps created near the leading-edge of the $2 / 3$ of the blade from tip;

- Scenario 3-26 bumps created at the half chord positions of the airfoils in the middle section of the blade;

- Scenario 4-26 bumps created near the trailing-edge of the tip section of the blade.

Herein, it is necessary to note that in order to ease the creation of the surface bumps, a constant distance between neighboring bumps, i.e., $0.05 \mathrm{~m}$, was defined to create the bumps on blade surface 
by using the 'revolve function' available in ANSYS workspace. Consequently, the numbers of bumps will be different when they are deployed in different areas of the blade. This is why different numbers of bumps are present in different bump array arrangement scenarios. The numerical blade models developed in the aforementioned four different bump array arrangement scenarios are shown in Figure 3. In the calculations of every bump array arrangement scenario, different tidal current speeds (i.e., $1.8,2$ and $3.2 \mathrm{~m} / \mathrm{s}$ ) and different angles of attack (i.e., $-5^{\circ}, 0^{\circ}, 5^{\circ}, 10^{\circ}$, and $15^{\circ}$ ) are considered, which allows us to understand the effects of the surface bumps on the efficiency of the blade at different tidal current speeds and angles of attack.

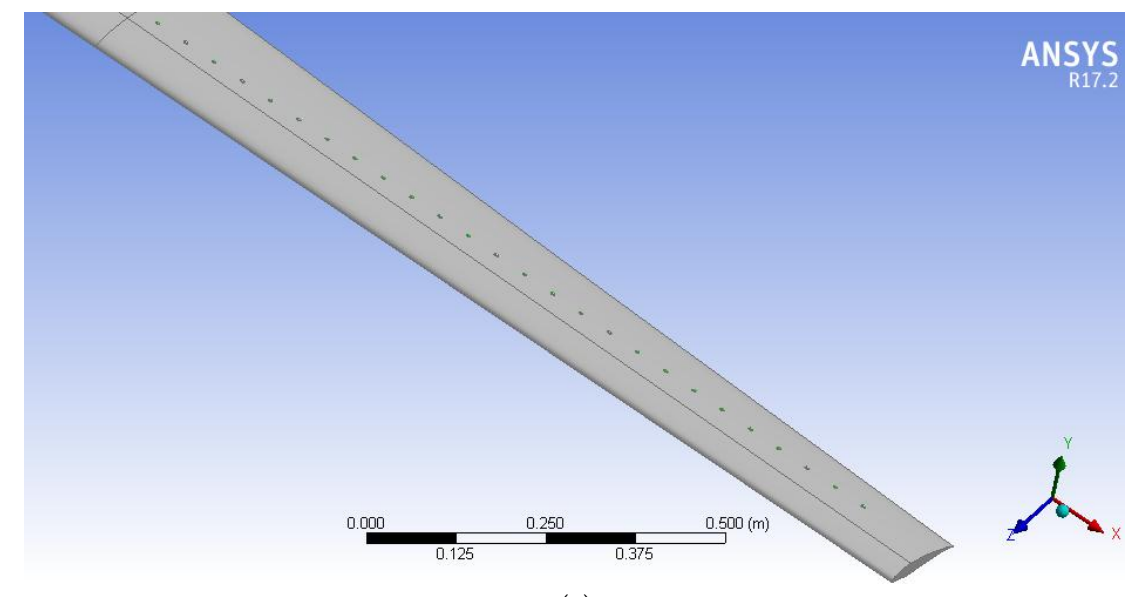

(a)

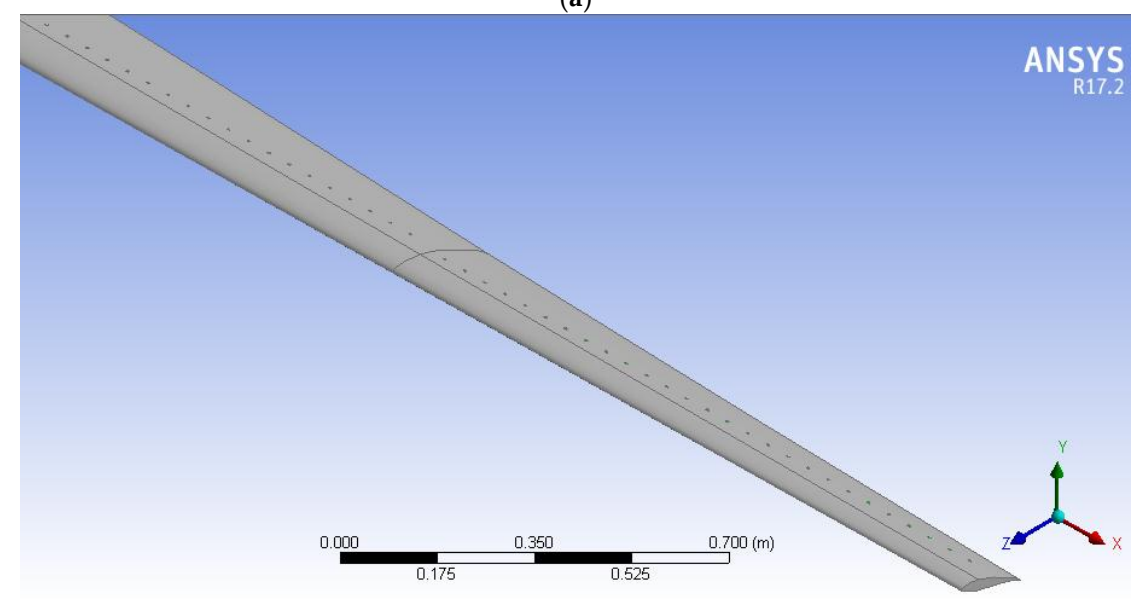

(b)

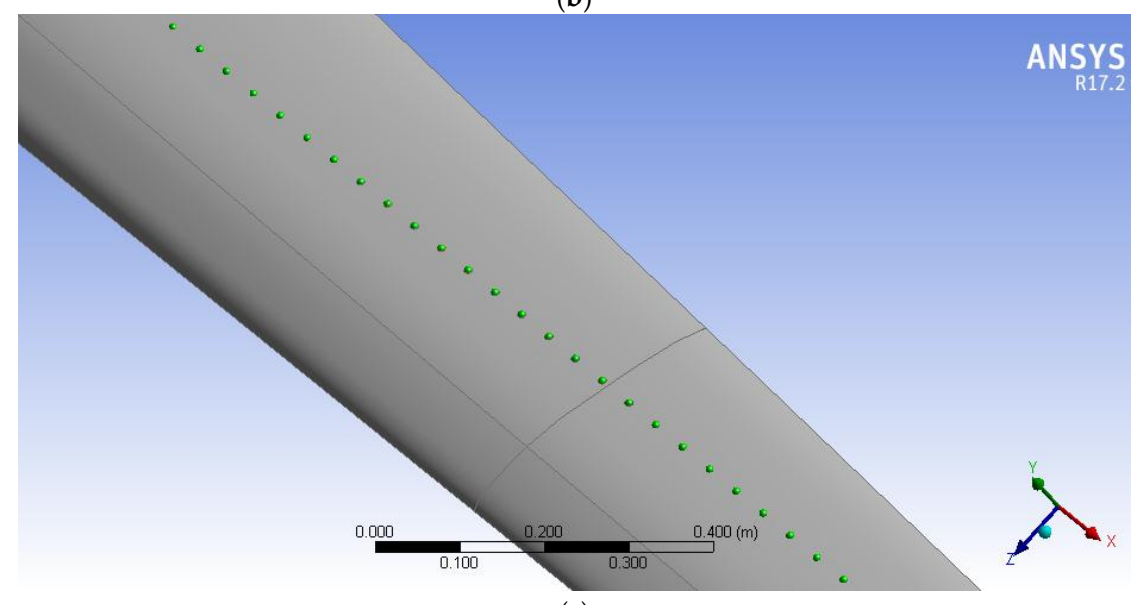

(c)

Figure 3. Cont. 


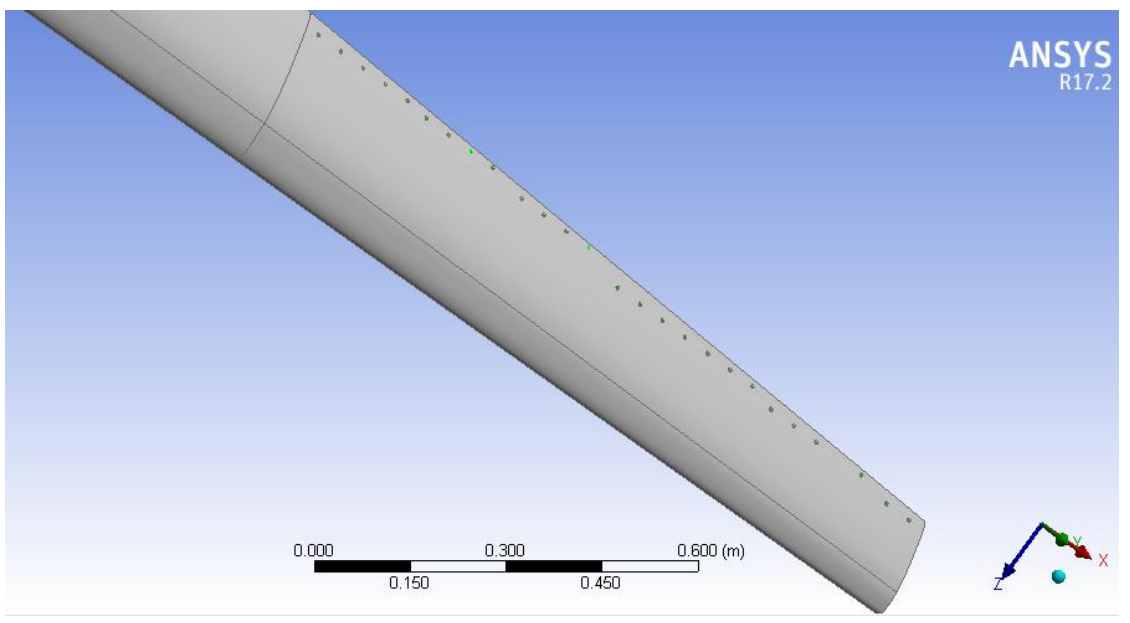

(d)

Figure 3. Different array arrangements of the surface bumps. (a) Scenario 1 (b) Scenario 2 (c) Scenario 3 (d) Scenario 4.

\section{Computational Setup}

In order to calculate the total lift and drag forces acting on the blade and therefore the rotor torque that is contributed by every blade, the numerical model of a three-bladed rotor is further developed based on blade models shown in Figure 3. In the rotor model, the three-bladed rotor is placed in the middle of a cylindrical fluid domain, as shown in Figure 4 . Where, the left and right circles and the dotted lines describe the size and boundaries of the fluid domain. The cyan-blue arrows indicate the flow direction in the domain. In the numerical calculations, the size of the cylindrical fluid domain is defined to be 5 times of the size of the rotor.

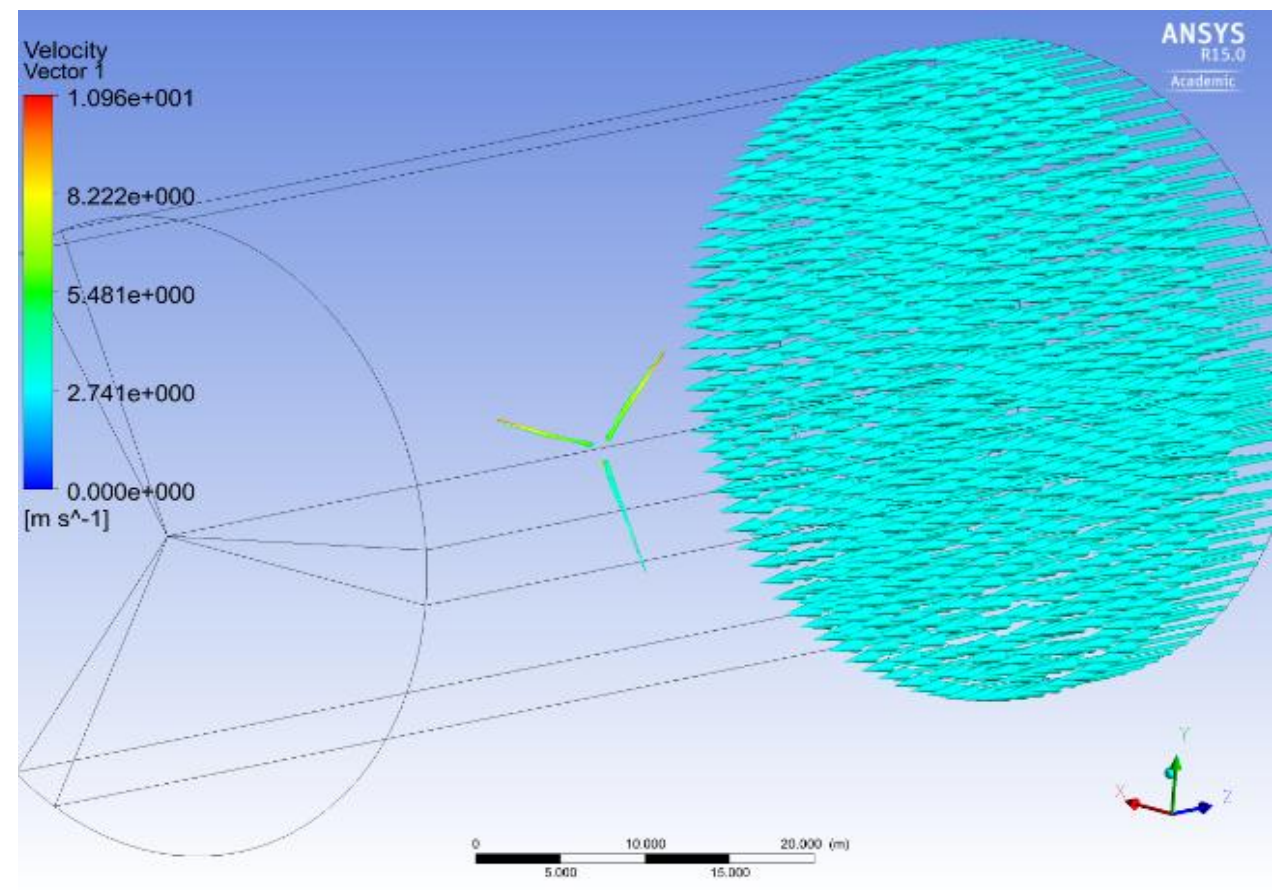

Figure 4. The numerical model of the three-bladed rotor in a cylindrical fluid domain. 
As the full cylindrical fluid domain in Figure 4 is very large in volume and the application of it will lead to a large amount of calculations. That is time consuming. Therefore, a simplified method is adopted in this paper to reduce the burden of calculation, i.e., only one-third division of the full cylindrical fluid domain is considered in the calculation. Since the velocity profile of tidal current is ignored (i.e., a constant current speed is assumed) and the three blades have the exactly same geometries, it is reasonable to believe that the three rotor blades are operating under identical conditions. The practice has shown that this is indeed an effective measure to reduce the burden of calculation while causing little unreliability in the calculation results. In the calculation, the fluid domain is designed as a rotating domain that rotates together with the blade rather than a stationary frame. Its boundary conditions are indicated in Figure 5.

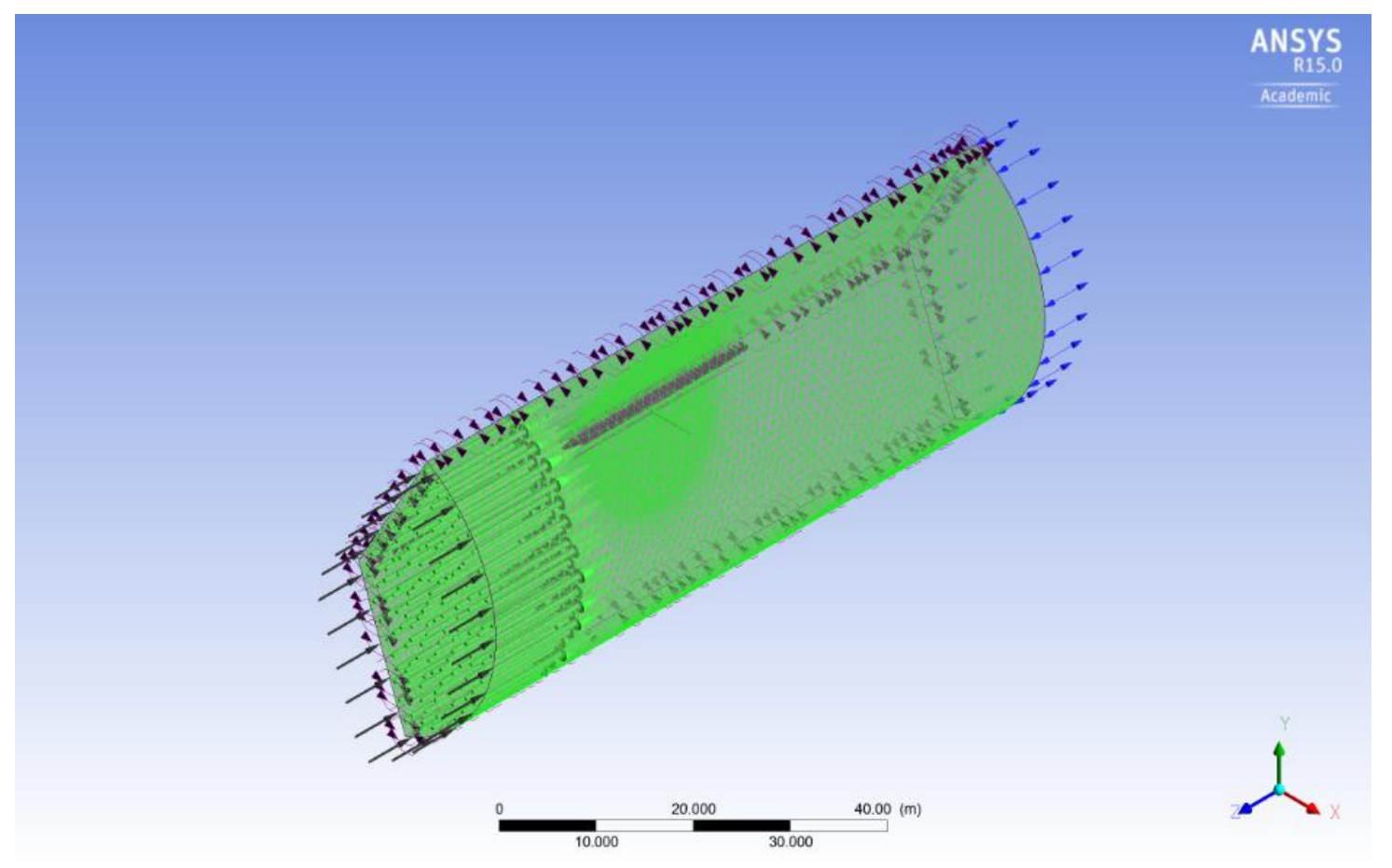

Figure 5. One third of the cylindrical fluid domain with boundary conditions.

In Figure 5, the purple arrows demonstrate the rotational periodicity connection of the interfaces. The bidirectional blue arrows on right side indicate the 'opening boundary', and those single directional black arrows on the left side show the 'inlet boundary'. It is worth noting that in the calculation, the 'inlet boundary' is defined as the normal speed with three velocity components in Cartesian Coordinates and the 'opening boundary' is defined as the opening pressure equivalent to 1.8 bar. The 'inlet boundary' is at an upstream distance of 5 rotor radius to the rotor, and the 'opening boundary' is at a downstream distance of 5 rotor radius away from the rotor. The medium of flow is seawater, of which the density is $1025 \mathrm{~kg} / \mathrm{m}^{3}$, viscosity $0.00108 \mathrm{Ns} / \mathrm{m}^{2}$, molecular weight $18.63 \mathrm{~g} / \mathrm{mole}$, and the temperature is $25^{\circ} \mathrm{C}$. Considering the Shear Stress Transport (SST) turbulence model is a hybrid model combining the K-omega and the K-epsilon models and it is, therefore, more accurate than the K-epsilon turbulence model [22,23], the SST turbulence model is adopted in the calculations presented in this paper.

In order to guarantee the reliability of the simulation results, in addition to the application of the default meshing technique that is provided by ANSYS, more measures are taken to refine the meshes of the model, especially in some specific areas of the domain (e.g., bumps, blade surfaces, etc.), and generate the meshes with acceptable mesh metric information. The measures include: 
- Match control—the two trapezoid faces were selected for the high and low geometry selections, respectively;

- Face sizing - the input blade sizing is $0.01 \mathrm{~m}$ and the behavior is set to be hard;

- Body sizing - a new coordinate system is used to precisely define and orient the location of the blade. Then, the sphere of influence control is utilized to create finer meshes around the blade with a radius of $10 \mathrm{~m}$ and $0.2 \mathrm{~m}$ as the minimum element size;

- Inflation-a local inflation is inserted around the blade with $0.01 \mathrm{~m}$ as the total thickness of the first inflation layer. The number of layers is selected to be 10, which means that 10 layers are defined within the inflation region, while the growth rate, i.e., the expansion ratio for each consecutive cell in the direction of the inflation was equal to 1.2;

- Advanced size function-the proximity and curvature is chosen and the fine option as the relevance center is enabled.

It was found that to mesh so complex a numerical model is quite time consuming. It is not realistic to assure the meshing quality by performing the mesh independency study as usual in a short time, let alone to identify the best meshing results in all four bump array arrangement scenarios by using such an approach. In order to resolve this issue, an alternative method was adopted in this paper to gauge the quality of meshing. It is the combined use of the minimum orthogonality and the maximum skewness of the generated meshes. Usually, the value of the minimum orthogonality is requested to be larger than 0.15 and the value of the maximum skewness is requested to be smaller than 0.95 . Take the model meshing in the scenario of the absence of bumps as an example, when the type of the element is defined as 'tet 4 ' the mesh metrics obtained after taking the aforementioned measures are shown in Figure 6.

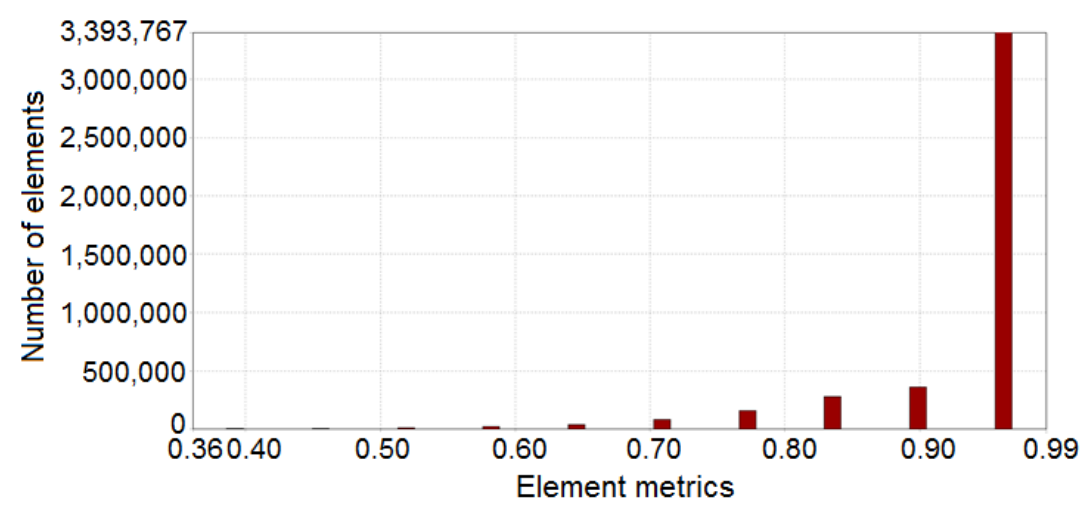

(a)

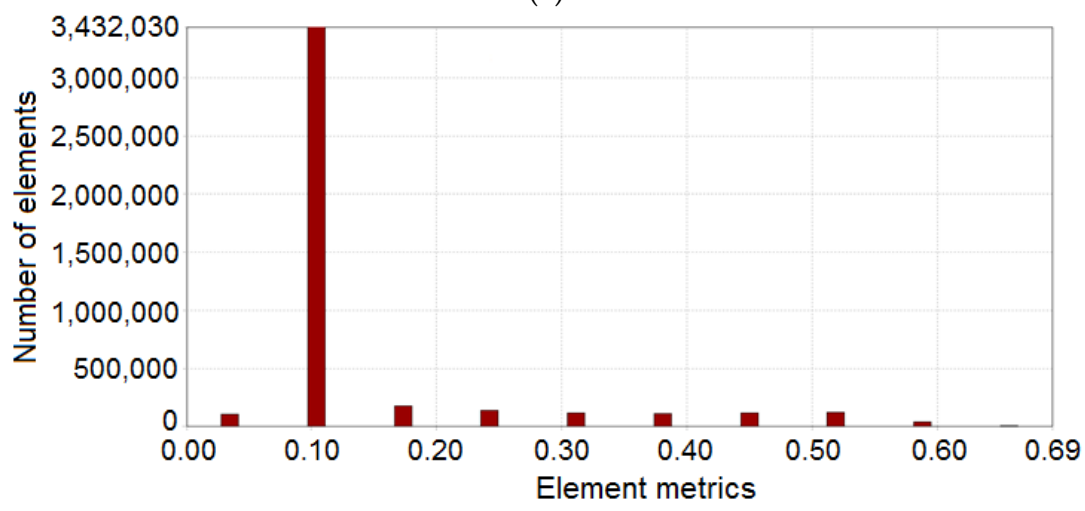

(b)

Figure 6. Mesh metrics obtained in the absence of bumps. (a) Orthogonal quality (b) Skewness. 
From Figure 6, it is found that the majority of elements have skewness of 0.10 and orthogonal quality of 0.97 . This implies that the meshing results obtained by using the above method is fully acceptable. This can, to a great extent, guarantee the reliability of the results obtained from simulation calculations. The corresponding tetrahedral meshing results are shown in Figure 7.

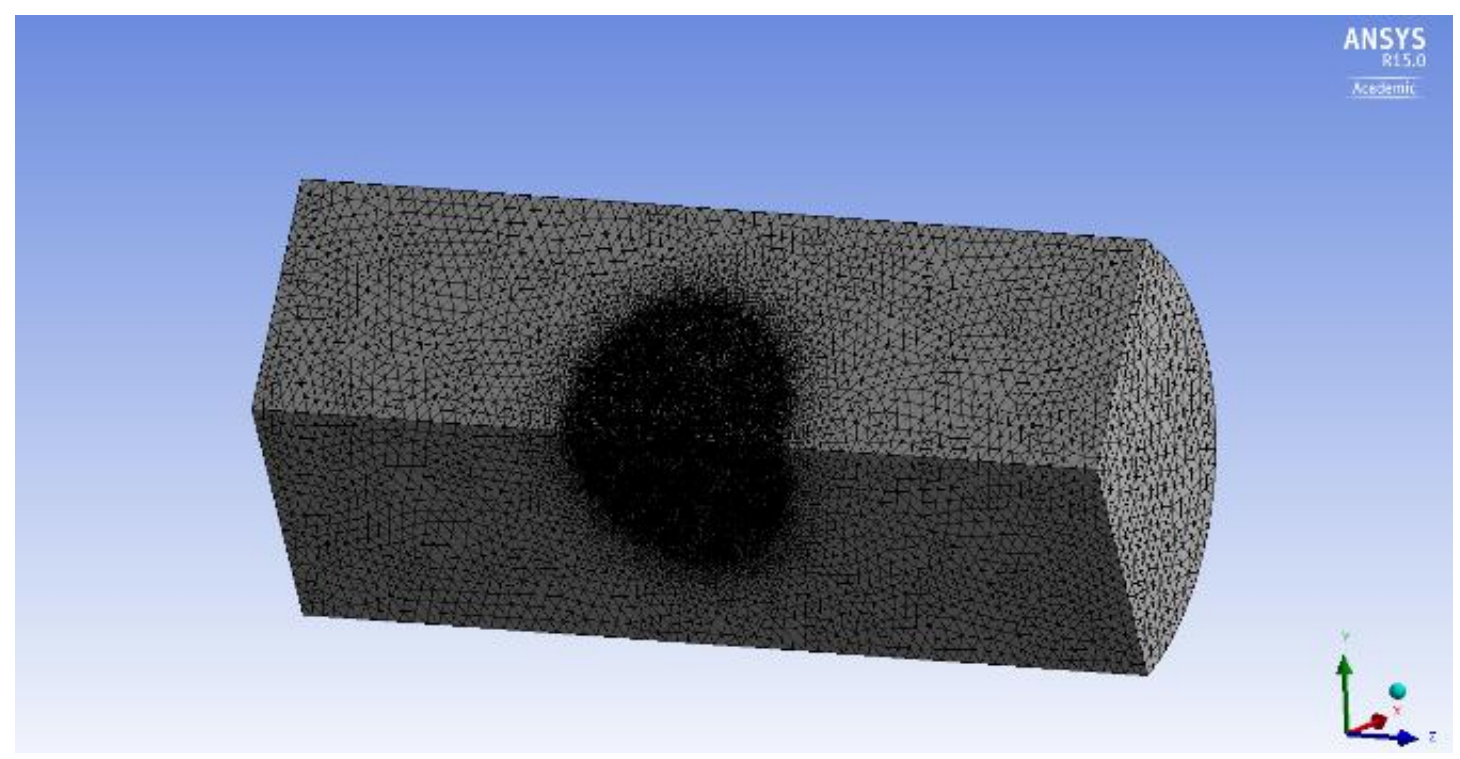

(a)

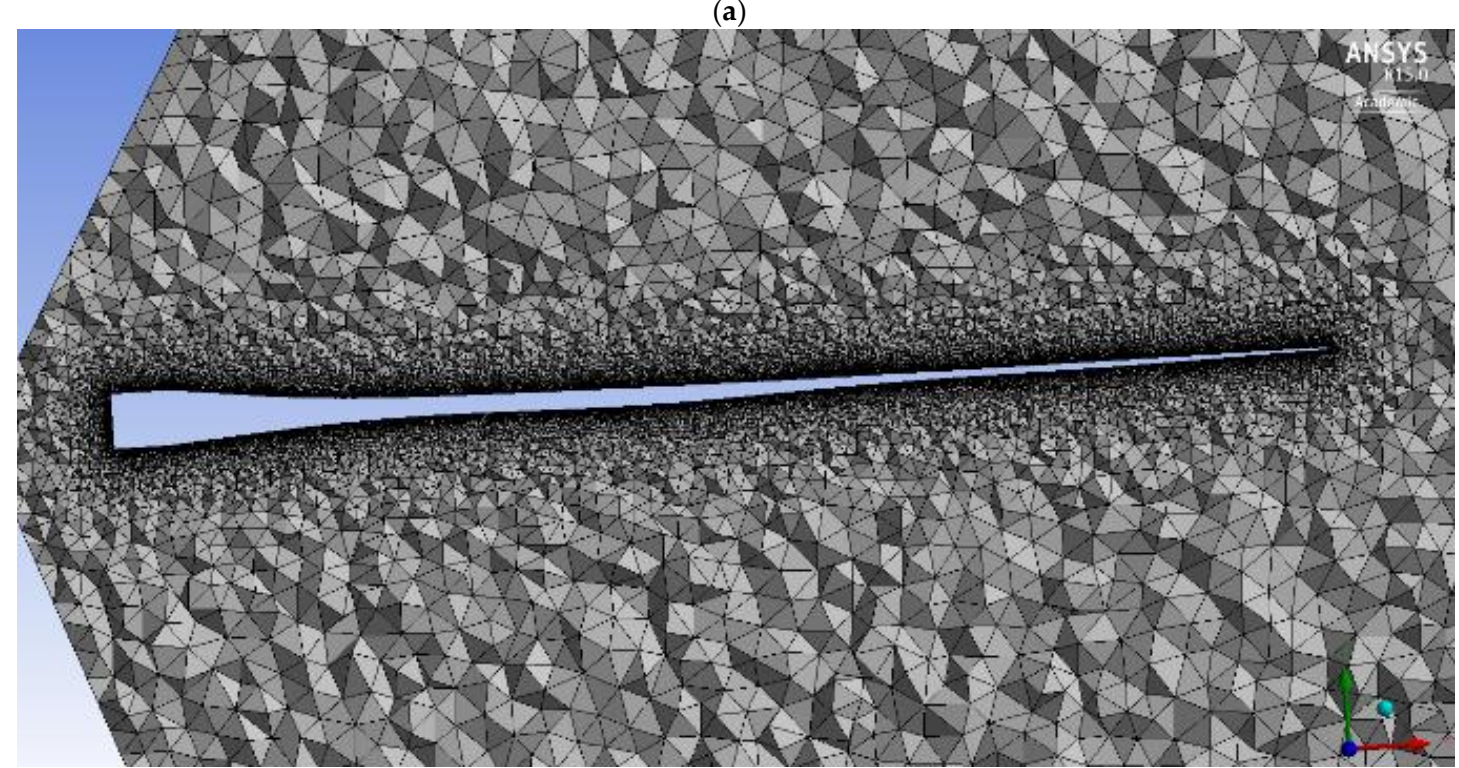

(b)

Figure 7. Tetrahedral meshing results in the absence of bumps. (a) Meshes in the whole domain (b) Meshes around the blade.

From Figure 7, it is seen that the cells located far from the blade have larger sizes, while those located close to the blade have smaller sizes. They correspond to $y^{+}$values ranging from 20 to 90 . As the presence of surface bumps will significantly increase the number of elements in the meshing results, the number of elements varies between 5 million and 9 million in the simulation calculations, depending on the designs and configurations of the bumps on the blade surface. 


\section{Numerical Calculations}

As mentioned earlier, three tidal current speeds, i.e., 1.8, 2 and $3.2 \mathrm{~m} / \mathrm{s}$, are considered in the following steady-state simulation calculations. The reason for selecting steady-state rather than transient simulation calculations to be performed in this paper is because the former is easier to converge, thus allows us to obtain desired results in a shorter time. Although this may somewhat sacrifice the simulation accuracy, the results from steady-state simulations are still able to basically predict the tendency of the change of interest. The tip speed ratio $\lambda$ of the blade can be calculated by

$$
\lambda=\frac{r \omega}{v}
$$

where $r$ and $\omega$ refer to the radius and angular speed of the turbine rotor, respectively; $v$ denotes the speed of tidal current.

Then, when the rotational speed of the turbine rotor is $8 \mathrm{rev} / \mathrm{min}$ the corresponding tip speed ratio $\lambda$ of the blade at these three tidal current speeds is calculated using (1). The resultant tip speed ratio $\lambda=3.26,2.93$ and 1.83 , respectively.

In order to understand the influence of the surface bumps on blade efficiency, the flow streamlines on the surface of the blade are calculated first at different tip speed ratios. Due to the calculation results obtained under different combination conditions of tip speed ratio, angle of attack and bump array arrangement are huge in number, it is unlikely to present all streamline calculation results within the limited context of the paper. For this reason, only the results obtained when the blade has no bumps and the angle of attack is $0^{\circ}$ are shown in Figure 8 as an illustrative example.

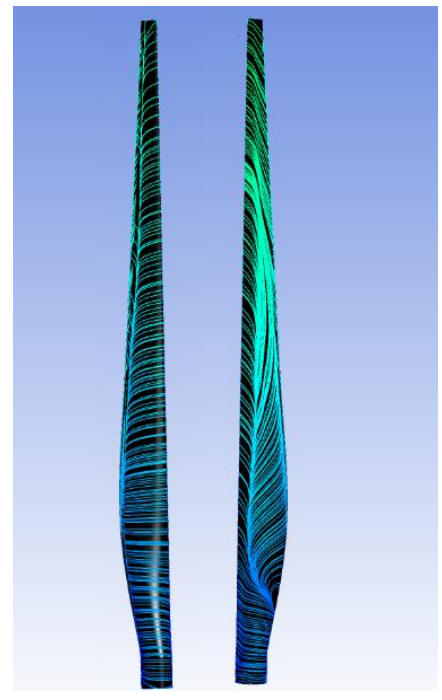

(a)

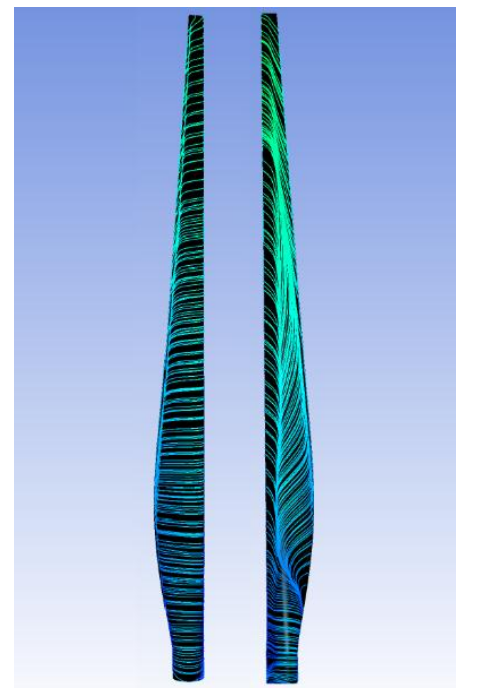

(b)



(c)

Figure 8. Streamlines on blade surface in the absence of bumps. (a) When $\lambda=1.83$ (b) When $\lambda=2.93$ (c) When $\lambda=3.26$.

From Figure 8, radial flow can be clearly observed from the surface of the rotating blade. Moreover, the higher the tip speed ratio, the stronger the radial flow will tend to be. Unfortunately, the further observation of the streamlines obtained in the presence of bumps discloses that the bump induced disturbances to the streamlines cannot be clearly observed from the calculated results. The reason might be due to either the tiny size of the bumps, the insufficient accuracy of the calculation results, or other unknown factors. However, it is well known that the surface roughness will affect the shedding of water flow from structure surface and the flow regime behind the structure [24], it is reasonable to believe that the application of the bumps on blade surface will definitely disturb the 
streamlines on blade surface and the flow regime behind the rotor. This explains why the surface bumps can potentially improve the energy capture efficiency of the blade.

Despite the failure to observe the disturbance of the bumps to the blade surface streamlines, the influences of the bumps on blade surface pressures and turbulence kinetic energy have been clearly observed from the calculation results. An illustrative example is given in Figure 9. As it is difficult to show all pressure calculation results obtained in all scenarios within the limited context of the paper, the maximum $P_{\max }$ and minimum pressures $P_{\min }$, obtained when the current speed is $3.2 \mathrm{~m} / \mathrm{s}$ and the attack angle is $0^{\circ}$, are listed in Table 2 as an example.

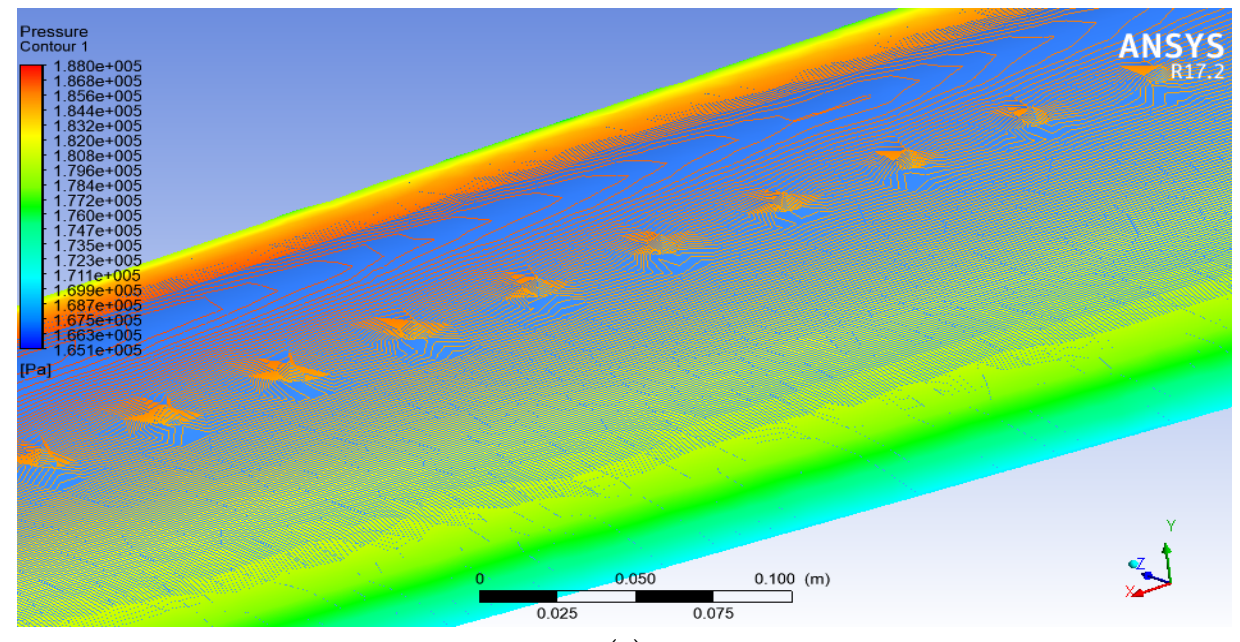

(a)

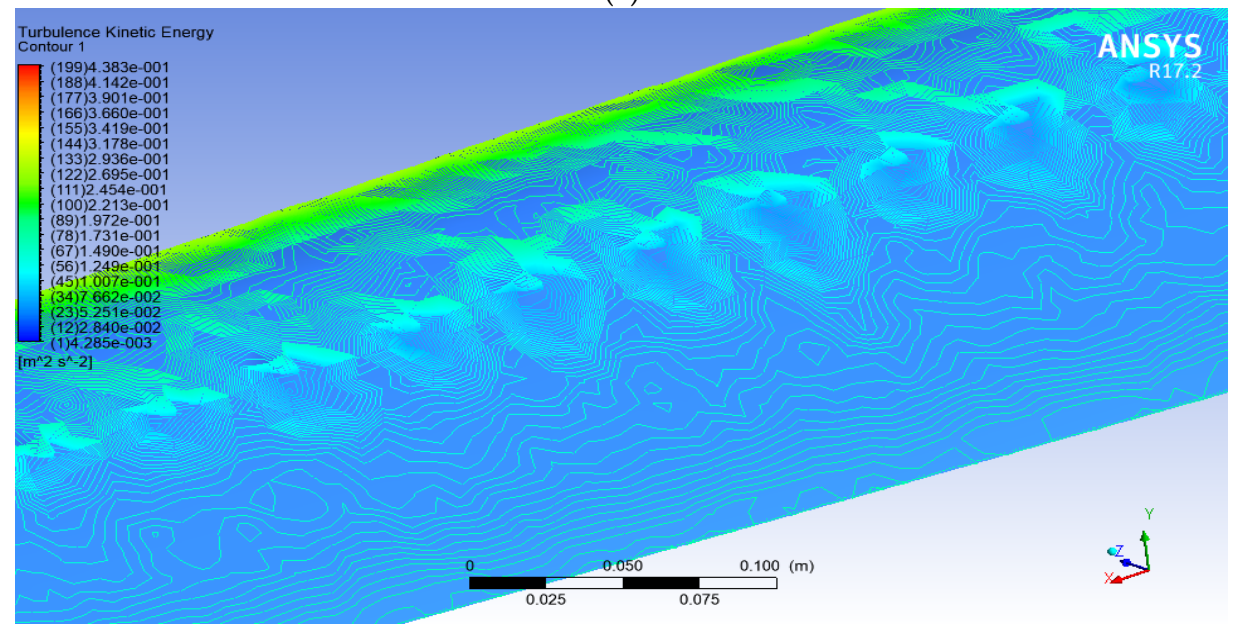

(b)

Figure 9. Disturbance resulted by the surface bumps. (a) Pressure (b) Turbulence kinetic energy.

Table 2. The $P_{\max }$ and $P_{\min }$ obtained when the current speed is $3.2 \mathrm{~m} / \mathrm{s}$ and pitch angle is $0^{\circ}$.

\begin{tabular}{ccc}
\hline Scenarios & $\boldsymbol{P}_{\min }(\mathbf{P a})$ & $\boldsymbol{P}_{\max } \mathbf{( P a )}$ \\
\hline Blade without bumps & 163,129 & 187,645 \\
Scenario 1 & 164,924 & 187,917 \\
Scenario 2 & 164,440 & 187,758 \\
Scenario 3 & 164,592 & 187,877 \\
Scenario 4 & 164,447 & 188,023 \\
\hline
\end{tabular}

From Table 2, it is seen that the application of the surface bumps does change the pressures on blade surface. Both the maximum pressure $P_{\max }$ and the minimum pressures $P_{\min }$ increase in the 
presence of the bumps, although the increments are different in different bump array arrangement scenarios. This suggests that the $P_{\max }$ and $P_{\min }$ on blade surface will increase as long as the bumps are present despite their array arrangement. Further research has shown that the $P_{\max }$ and $P_{\min }$ will increase as long as the bumps are present also despite the current speed and angle of attack. In order to illustrate this finding, the $P_{\max }$ and $P_{\min }$ in Scenario 1 and those obtained in the absence of the bumps are listed in Table 3.

Table 3. The $P_{\max }$ and $P_{\min }$ in scenario 1 and those obtained in the absence of the bumps.

\begin{tabular}{cccccc}
\hline & \multicolumn{2}{c}{ Blade without Bumps } & \multicolumn{2}{c}{ Scenario 1 } \\
\cline { 3 - 6 } & & $\boldsymbol{P}_{\min }(\mathbf{P a})$ & $\left.\boldsymbol{P}_{\text {max }} \mathbf{( P a}\right)$ & $\boldsymbol{P}_{\min }(\mathbf{P a})$ & $\boldsymbol{P}_{\max } \mathbf{( P a )}$ \\
\hline \multirow{3}{*}{ Current speed } & $1.8 \mathrm{~m} / \mathrm{s}$ & 162,339 & 188,432 & 163,804 & 188,602 \\
& $2 \mathrm{~m} / \mathrm{s}$ & 162,385 & 188,367 & 163,961 & 188,515 \\
& $3.2 \mathrm{~m} / \mathrm{s}$ & 163,129 & 187,645 & 164,924 & 187,917 \\
Angle of attack & $-5^{\circ}$ & 162,832 & 187,647 & 165,045 & 187,704 \\
& $5^{\circ}$ & 162,476 & 188,090 & 164,772 & 188,160 \\
& $10^{\circ}$ & 162,287 & 188,339 & 164,609 & 188,413 \\
& $15^{\circ}$ & 162,076 & 188,609 & 164,439 & 188,669 \\
\hline
\end{tabular}

From Table 3, it is seen that the $P_{\max }$ and $P_{\min }$ on blade surface do increase as long as the bumps are present. Moreover, the same phenomenon can always be observed whatever the values of the current speed and the angle of attack are. Since the change in pressure distribution will lead to the change in pressure gradient, it is sure that the lift force $F_{L}$ and drag force $F_{D}$ acting on the blade and the rotor torque $T$ contributed by every blade will be affected by the bumps as well. In order to prove this prediction, the corresponding $F_{L}, F_{D}$ and $T$ obtained when the current speed is $3.2 \mathrm{~m} / \mathrm{s}$ and the attack angle is $0^{\circ}$ are listed in Table 4 . In the calculation, the bump size is still $5 \mathrm{~mm}$.

Table 4. The $F_{L}$ and $F_{D}$ acting on the blade.

\begin{tabular}{cccccc}
\hline & Blade without Bumps & Scenario 1 & Scenario 2 & Scenario 3 & Scenario 4 \\
\hline$F_{L}(\mathrm{~N})$ & $19,245.10$ & $19,175.70$ & $19,376.70$ & $19,701.3$ & $19,433.40$ \\
$F_{D}(\mathrm{~N})$ & 6289.76 & 6176.33 & 6319.28 & 6351.67 & 6251.44 \\
$F_{L} / F_{D}$ & 3.0600 & 3.1050 & 3.0660 & 3.1020 & 3.1090 \\
$T(\mathrm{~N} \cdot \mathrm{m})$ & $95,220.20$ & $95,538.60$ & $96,312.60$ & $97,713.40$ & $96,577.00$ \\
\hline
\end{tabular}

In order to facilitate the analysis, the ratio of $F_{L} / F_{D}$ and the consequent torque $T$ obtained in all scenarios are also graphically shown in Figure 10.

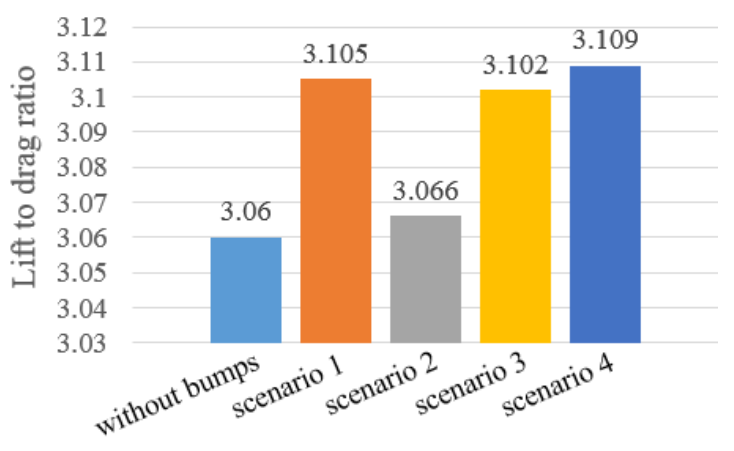

(a)

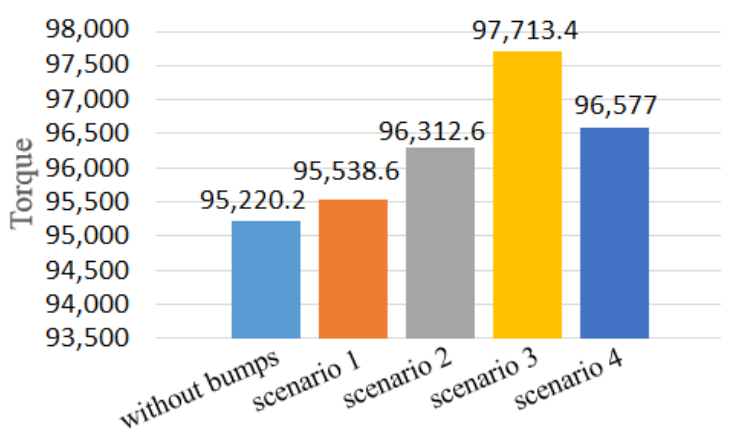

(b)

Figure 10. The influence of surface bumps on the lift and drag forces and rotor torque. (a) Ratio of $F_{L} / F_{D}(\mathbf{b})$ Torque $T$. 
From Figure 10, it is seen that the application of surface bumps does improve the ratio of lift to drag forces and enhance the contribution of every blade to the rotor torque. However, it is noticed that the contributions of the surface bumps to the $F_{L} / F_{D}$ and $T$ are different in different scenarios, i.e., improving the ratio of $F_{L} / F_{D}$ by $0.2 \sim 1.6 \%$ and improving torque $T$ by $0.3 \sim 2.6 \%$. This suggests that the contribution of the bumps can be further improved through optimizing their array arrangement on the surface of the blade.

Subsequently, the influences of the surface bumps on the $F_{L} / F_{D}$ at other tidal current speeds and angles of attack are investigated. The corresponding results obtained in scenario 1 and those obtained from the blade without bumps are shown in Figures 11 and 12.

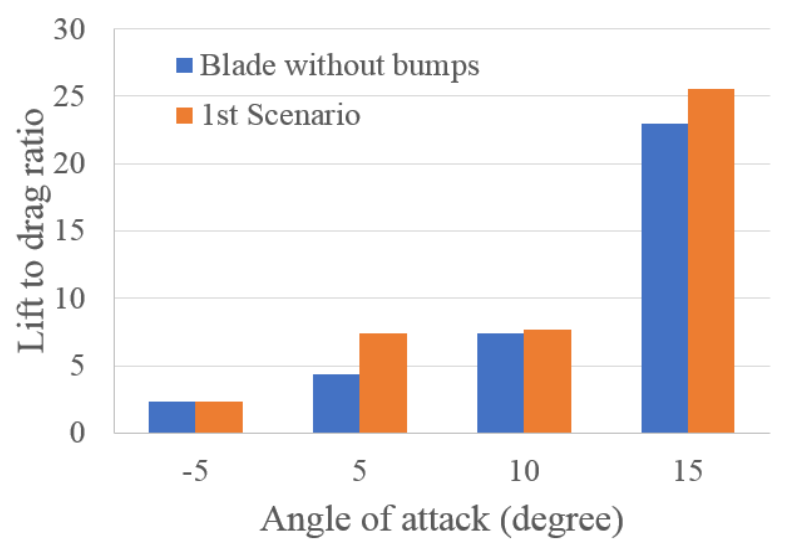

Figure 11. Influences of the bumps on $F_{L} / F_{D}$ at different angles of attack.

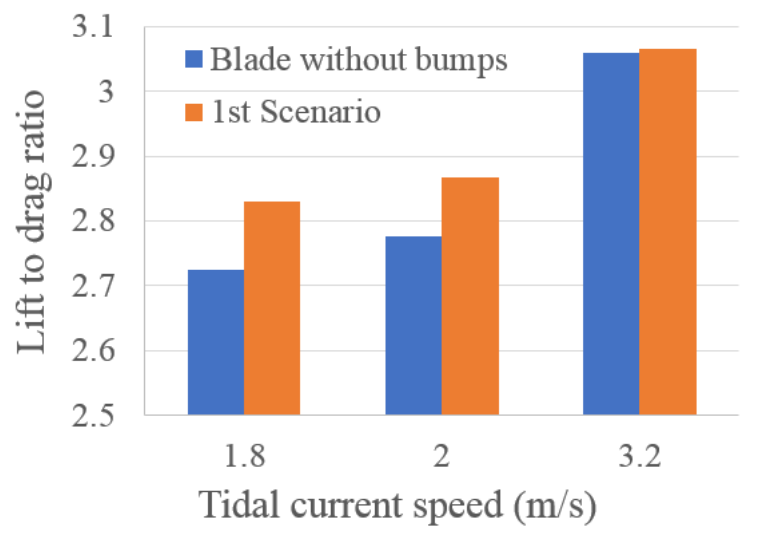

Figure 12. Influences of the bumps on $F_{L} / F_{D}$ at tidal current speeds.

From Figure 11, it is clearly seen that when the angle of attack increases from $-5^{\circ}$ to $15^{\circ}$, the corresponding value of $F_{L} / F_{D}$ increases gradually whatever the presence or absence of the surface bumps. Through comparing the $F_{L} / F_{D}$ results obtained in every scenario before and after the application of the bumps, it is found that when the bumps are present the value of $F_{L} / F_{D}$ is more or less increased in all four cases of angle of attack.

Similarly, from Figure 12 it is found that when the tidal current speed increases from 1.8 to $3.2 \mathrm{~m} / \mathrm{s}$, the corresponding value of $F_{L} / F_{D}$ also increases whatever the presence or absence of the bumps. Through comparing the results of $F_{L} / F_{D}$ obtained in every scenario before and after the bumps are applied, it is found that after the bumps are present the value of $F_{L} / F_{D}$ is increased to certain extent in all three cases of tidal current speed. 
In summary, the phenomena shown in Figures 11 and 12 suggest that the surface bumps do play a positive role in improving the ratio of the lift to drag forces acting on the blade and moreover they always work effectively in spite of the angle of attack and tidal current speed.

\section{Conclusions}

Aiming at exploring an effective measure that can be applied to improve the energy capture efficiency of both new and existing tidal turbine blades, a numerical research is conducted systematically in this paper with the aid of ANSYS CFX. The research is focused on investigating the influences of the surface biomimetic features on the blade surface pressure and the ratio of the lift to drag forces acting on the blade under different combination conditions of bump array arrangement, angle of attack, and tidal current speed. Through analyzing the numerical calculation results obtained in different scenarios, the following two conclusions can be drawn:

(1) Despite the failure of observing the bump effect on the blade surface streamlines, the influences of the bumps on blade surface pressure and turbulence kinetic energy are clearly observed. The values of $P_{\max }$ and $P_{\min }$ indicate that both the maximum and the minimum pressures on blade surface will increase as long as the bumps are present on blade surface in spite of the tidal current speed, the angle of attack, and bump array arrangement;

(2) Surface bumps do improve the ratio of the lift to drag forces acting on the blade thus the torque created by the turbine rotor. Moreover, they always work effectively in spite of the bump array arrangement, the angle of attack and tidal current speed. The simulation results have shown that when the current speed is $3.2 \mathrm{~m} / \mathrm{s}$ and the attack angle is $0^{\circ}$, the ratio of $F_{L} / F_{D}$ can be improved by $0.2 \sim 1.6 \%$ and the rotor torque $T$ can be improved by $0.3 \sim 2.6 \%$. Since the contribution of the surface bumps to the improvement of $F_{L} / F_{D}$ and $T$ is different in different array arrangement scenarios, the contribution of the bumps can be further improved through optimizing their array arrangement on the surface of the blade.

Following the work reported above, the size effect, the influences of the geometric shape and the number of biomimetic features on the energy capture efficiency of the blade, as well as the position to apply the biomimetic features will be further researched in the future in order to achieve an optimal design of the surface biomimetic technique. The achievements will be reported in a separate paper.

Acknowledgments: The work reported in this paper is supported by the UK EPSRC funding with the reference number of EP/R021503/1.

Author Contributions: Wenxian Yang interpreted the numerical calculation results and wrote the paper; Theodoros Alexandridis and Wenye Tian developed the numerical models of the tidal current turbine blade and performed the numerical calculations in different scenarios.

Conflicts of Interest: The authors declare no conflict of interest.

\section{References}

1. Mofor, L.; Goldsmith, J.; Jones, F. Ocean Energy: Technology Readiness, Patents, Deployment Status and Outlook, International Renewable Energy Agency (IRENA) Report, Paris, August 2014. Available online: http:/ / www.irena.org/DocumentDownloads/Publications/IRENA_Ocean_Energy_report_2014. pdf (accessed on 9 March 2018).

2. Charlie, R.H.; Justus, J.R. Ocean Energies: Environmental, Economic and Technological Aspects of Alternative Power Sources; Elsevier: Amsterdam, The Netherlands, 1993.

3. World Energy Resources: Marine Energy, World Energy Council Report, 2016. Available online: http: //www.worldenergy.org/wp-content/uploads/2017/03/WEResources_Marine_2016.pdf (accessed on 9 March 2018).

4. Corsatea, T.D.; Magagna, D. Overview of European Innovation Activities in Marine Energy Technology, JRC Science and Policy Reports, 2014. Available online: http://setis.ec.europa.eu/sites/default/files/ reports/Ocean-Energy-Innovation.pdf (accessed on 9 March 2018). 
5. Lewis, M.; Neill, S.P.; Robins, P.; Hashemi, M.R.; Ward, S. Characteristics of the velocity profile at tidal-stream energy sites. Renew. Energy 2017, 114 Pt A, 258-272. [CrossRef]

6. Hau, E. Wind Turbines: Fundamentals, Technologies, Applications, Economics, 2nd ed.; Springer: Berlin, Germany, 2006.

7. Chattot, J.J. Optimization of wind turbines using helicoidal vortex model. J. Sol. Energy Eng. Trans. ASME 2003, 125, 418-424. [CrossRef]

8. Duquette, M.M.; Visser, K.D. Numerical implications of solidity and blade number on rotor performance of horizontal-axis wind turbines. J. Sol. Energy Eng. Trans. ASME 2003, 125, 425-432. [CrossRef]

9. Gasch, R.; Twele, J. Wind Power Plants; Solarpraxis: Berlin, Germany, 2002.

10. Schubel, P.J.; Crossley, R.J. Wind turbine blade design. Energies 2012, 5, 3425-3449. [CrossRef]

11. Fish, F.E.; Battle, J.M. Hydrodynamic design of the humpback whale flipper. J. Morphol. 1995, 225, 51-60. [CrossRef] [PubMed]

12. Fish, F.E.; Weber, P.W.; Murray, M.M.; Howle, L.E. The tubercles on humpback whales' flippers: Application of bio-inspired technology. Integr. Comp. Biol. 2011, 51, 203-213. [CrossRef] [PubMed]

13. Yan, R.D. Tubercle Leading Edges. Master's Thesis, Imperial College London, London, UK, 2015.

14. Shi, W.; Rosli, R.; Atlar, M.; Norman, R.; Wang, D.; Yang, W. Hydrodynamic performance evaluation of a tidal turbine with leading-edge tubercles. Ocean Eng. 2016, 117, 246-253. [CrossRef]

15. Kulkarni, S.S.; Chapman, C.; Shah, H.; Parn, E.A.; Edwards, D.J. Design study of horizontal axis tidal turbine blade. Mindanao J. Sci. Technol. 2017, 15, 12-34.

16. Alexandridis, T. Numerical Research of Innovative Biomimetic Concept of Tidal Turbine Blades. Master's Thesis, Newcastle University, Newcastle upon Tyne, UK, 2017.

17. Tsai, K.C.; Pan, C.T.; Cooperman, A.M.; Johnson, S.J.; Dam, C.P. An innovative design of a microtab deployment mechanism for active aerodynamic load control. Energies 2015, 8, 5885-5897. [CrossRef]

18. Fernandez-Gamiz, U.; Zulueta, E.; Boyano, A.; Ansoategui, I.; Uriarte, I. Five megawatt wind turbine power output improvements by passive flow control devices. Energies 2017, 10, 742. [CrossRef]

19. Fernandez-Gamiz, U.; Zulueta, E.; Boyano, A.; Ramos-Hernanz, J.A.; Lopez-Guede, J.M. Microtab design and implementation on a 5 MW wind turbine. Appl. Sci. 2017, 7, 536. [CrossRef]

20. Aramendia, I.; Fernandez-Gamiz, U.; Antonio Ramos-Hernanz, J.; Sancho, J.; Manuel Lopez-Guede, J.; Zulueta, E. Chapter 21: Flow control devices for wind turbines. In Energy Harvesting and Energy Efficiency; Bizon, N., Tabatabaei, N.M., Blaabjerg, F., Kurt, E., Eds.; Springer: New York, NY, USA, 2017.

21. Lachance-Barrett, S.; Corona, E. Wind Turbine Blade FSI (Part 1)—Geometry. Available online: http: / / confluence.cornell.edu/display /SIMULATION/Wind+Turbine+Blade+FSI+\%28Part+1\% 29+-+Geometry (accessed on 10 March 2018).

22. Menter, F.R. Zonal two equation Kappa-Omega turbulence models for aerodynamic flows. In Proceedings of the 24th AIAA Fluid Dynamics Conference, Orlando, FL, USA, 6-9 July 1993.

23. Menter, F.R. Two-equation eddy-viscosity turbulence models for engineering applications. AIAA J. 1994, 32, 1598-1605. [CrossRef]

24. Bengt, S. Vortex Shedding. Available online: http://www.thermopedia.com/content/1247 (accessed on 11 March 2018).

(C) 2018 by the authors. Licensee MDPI, Basel, Switzerland. This article is an open access article distributed under the terms and conditions of the Creative Commons Attribution (CC BY) license (http://creativecommons.org/licenses/by/4.0/). 